\title{
PROFESSOREN UND IHRE NEBENTÄTIGKEITEN ANHAND DER VITEN VON MELCHIOR ADAM - KARRIEREN AUSSERHALB DER UNIVERSITÄT
}

\author{
KLAUS-DIETER BEIMS
}

\author{
PROFESSORS AND THEIR SECONDARY EMPLOYMENT ACCORDING \\ TO THE WORK VITAE (LIVES) BY MELCHIOR ADAM - CAREERS OUTSIDE UNIVERSITY
}

The paper presents the typical course of education, life journeys, and careers of professors of theology and law based on selected Viten (Vitae, biographies) from an early-modern collection of biographies by the Heidelberg historian Melchior Adam (1575-1622), who was originally from Silesia. First and foremost, it attempts to determine the degree to which professors were involved in non-university structures: theologians as priests, superintendents, etc., jurists as princes or town councillors, etc. It also examines the role played by graduation from and study at foreign schools in their future university and other careers.

Keywords: Melchior Adam - Johannes Bugenhagen - Justus Jonas - David Chytraeus - biographies - early modern period - professorial careers - professors of theology - professors of law - University of Wittenberg University of Rostock

DOI: $10.14712 / 23365730.2020 .31$

Gegenstand meines Aufsatzes ist die zu Anfang des 17. Jahrhunderts erschienene Sammlung von Gelehrtenbiographien des aus Schlesien stammenden Humanisten Melchior Adam (1575-1622). Moderne Forschung zu frühneuzeitlichen Biographiensammlungen ${ }^{1}$ nähert sich diesem Thema oft unter der Fragestellung, wie sich in den Viten die Herausbildung und Darstellung von Individualität vollzieht und in welcher Weise in ihnen Einzelpersönlichkeiten narrativ konstruiert bzw. konstruiert werden. ${ }^{2}$ Insbesondere wird in den Blick genommen, wie sich biographisches Schreiben unter den Vorzeichen von Reformation,

1 Vgl. den einführenden Überblick zu Stand und Tendenzen der Forschung: Karl EnEnKEL - Claus ZitTEL, Einleitung, in: Karl Enenkel - Claus Zittel (Hgg.): Die Vita als Vermittlerin von Wissenschaft und Werk. Formund Funktionsanalytische Untersuchungen zu frühneuzeitlichen Biographien von Gelehrten, Wissenschaftlern, Schriftstellern und Künstlern, Berlin 2013, S. 1-8.

2 Vgl. dazu Karl A. Enenkel, Die Erfindung des Menschen. Die Autobiographik des frühneuzeitlichen Humanismus von Petrarca bis Lipsius, Berlin - New York 2008; Karl ENENKEL - Betsy DE Jong-CRANE Peter Liebregts (Hgg.), Modelling the Individual. Biography and Portrait in the Renaissance, Amsterdam Atlanta 1998; Christian von Zimmermann, Biographik und Individualität. Überlegungen zum Problemhorizont biographischer Schreibformen, in: Andreas Schüle (Hg.): Biographie als religiöser und kultureller Text / Biography as a religious and cultural text, Münster 2002, S. 21-40. 
Gegenreformation und Konfessionalisierung vollzog. ${ }^{3}$ Des Weiteren galt das Interesse den Einflüssen des Humanismus auf die frühneuzeitliche Biographik. ${ }^{4}$

Wenn vom Konstruktcharakter der Viten die Rede ist, bedeutet das, dass es in ihnen nicht primär um wahrheitsgetreue Lebensbilder von Individuen und Überprüfbarkeit der mitgeteilten Fakten im Sinne heutiger Historiographie geht. Der Mensch wird, wie es Karl A. Enenkel in seiner monumentalen Studie zur Autobiographik der Renaissance, Die Erfindung des Menschen, formuliert hat, in den Viten nicht dokumentarisch abgebildet, sondern durch die Literatur konstituiert. ${ }^{5}$ Und was eine der primären Quellen Adams, die Leichenpredigten, betrifft, hat Thomas Kaufmann in seiner Untersuchung der Biographien Rostocker Professoren bemerkt, „... daß es in ihnen nicht um die Darstellung des Individualitätswertes einer Person geht, sondern um konventionierte Exempel christlicher Amtsführung in Schule und Universität, Kirche und Familie. "6 Und zur Darstellung von Glauben, Lebensvollzug und Amtspraxis: Die Darstellungen präsentieren einen „typus fidelium“, „Die individuelle Person tritt ganz hinter ihre ethisch-religiöse Exemplarität zurück. "7

Der vorliegende Aufsatz geht mit einem das Individuum übergreifenden methodischen Ansatz an eine solche frühneuzeitliche Vitensammlung heran. Dieser geht davon aus, dass die einzelnen Viten einer solchen Sammlung aufgrund ihrer Quellengrundlage ${ }^{8}$ und ihres paratextuellen Status ${ }^{9}$ eben nicht nur konstruierte Persönlichkeitsbilder im Sinne einer positiven memoria eines Individuums oder bestimmter normativer Ideale, denen die dargestellte Person zu entsprechen hatte, liefern, sondern auch eine Fülle von Informationen zu Wissens- und Wissenschaftsfeldern sowie belastbare prosopographische Details zu einzelnen Personen und Personengruppen. Insofern stellt eine Vitensammlung wie diejenige Adams

3 Vgl. dazu Irena Backus, Life Writing in Reformation Europe. Lives of Reformers by Friends, Disciples and Foes, Aldershot 2008; Herbert ImmenkötтеR, Von Engeln und Teufeln: Über Luther-Biographien des 16. Jahrhunderts, in: August Buck (Hg.), Biographie und Autobiographie in der Renaissance, Wiesbaden 1983, S. 91-102; Robert KolB, Burying the Brethren: Lutheran Funeral Sermons as Life-Writing, in: Thomas F. Mayer - D. R. Woolf (Hgg.), The Rhetorics of Life-Writing in Early Modern Europe. Form of Biography from Cassandra Fedele to Louis XIV, 4. Aufl., Ann Arbor 1998, S. 97-113; Matthias PohLIG, Zwischen Gelehrsamkeit und konfessioneller Identitätsstiftung. Lutherische Kirchen- und Universalgeschichtsschreibung 1546-1617, Tübingen 2007; Andreas SchüLE (Hg.), Biographie als religiöser und kultureller Text / Biography as a religious and cultural text, Münster 2002; Günther WARTENBERG, Melanchthonbiographien vom 16. bis zum 19. Jahrhundert, in: Günther Wartenberg (Hg.), Werk und Rezeption Philipp Melanchthons in Universität und Schule bis ins 18. Jahrhundert, Leipzig 1999, S. 179-194; Timothy J. Wengert, “With Friends Like This ...": The Biography of Philip Melanchthon by Joachim Camerarius, in: T. F. Mayer - D. R. Woolf (Hgg.), The Rhetorics of Life-Writing in Early Modern Europe, S. 115-131; Eike Wolgast, Biographie als Autoritätsstiftung: Die ersten evangelischen Lutherbiographien, in: Walter Berschin (Hg.), Biographie zwischen Renaissance und Barock. Zwölf Studien, Heidelberg 1993, S. 41-71.

4 Vgl. dazu Walter Berschin (Hg.), Biographie zwischen Renaissance und Barock. Zwölf Studien, Heidelberg 1993; August Buck (Hg.), Biographie und Autobiographie in der Renaissance, Wiesbaden 1983; Marc Fumaroli, From „Lives“ to Biography: The Twilight of Parnassus, Diogenes 239, 1987, S. 1-27; James M. WeIss (Hg.), Humanist Biography in Renaissance Italy and Reformation Germany. Friendship and Rhetotic, Farnham - Burlington 2010.

5 K. ENENKel, Die Erfindung des Menschen, S. 13.

6 Thomas Kaufmann, Universität und lutherische Konfessionalisierung. Die Rostocker Theologieprofessoren und ihr Beitrag zur theologischen Bildung und kirchlichen Gestaltung im Herzogtum Mecklenburg zwischen 1550 und 1675, Heidelberg 1997, S. 139.

7 T. Kaufmann, Universität und lutherische Konfessionalisierung, S. 166.

8 Viele Viten Adams basieren auf Memorialtexten wie Leichenpredigten, akademischen Gedenkreden etc., die immer auch einen umfangreichen biographischen Teil enthielten.

9 Viele Viten Adams beruhten auf Viten in Werkausgaben und wurden später ihrerseits wieder in entsprechender Weise verwendet. 
nicht nur eine gute Quelle für die Wissens- und Wissenschaftsgeschichte der frühen Neuzeit dar, sondern bietet auch eine hervorragende Basis dafür, vergleichend die Rahmenbedingungen für Wissenschaftlerkarrieren und die grundlegenden Konstituenten der gesellschaftlichen Rolle und Funktion bestimmter Gruppen von Wissenschaftlern in diesem Zeitraum herauszuarbeiten. Abgesehen davon bietet eine solche Vitensammlung, gerade wenn man die Überformung der Darstellung anhand normativer Ideale in Rechnung stellt, auch interessante mentalitätsgeschichtliche Einblicke in Denkvorstellungen und Verhaltensnormen bestimmter gesellschaftlicher Gruppen in dieser Zeit.

Meine kleine, sehr selektive Reise durch die Gelehrtenwelt des 16. Jahrhunderts beginnt um 1600 im reformierten Heidelberg. Melchior Adam war 1598 nach Heidelberg gekommen und wurde dort Schulleiter des Paedagogium. Während seiner vorhergehenden Schullaufbahn war Adam mit calvinistischen Positionen in Berührung gekommen: Geboren 1575 im schlesischen Grottkau, besuchte er ab 1589 das Gymnasium in nahegelegenen Brieg - eine Schule, an der der streng lutherische Herzog Georg II. von Brieg bereits seit 1575 kryptocalvinistische Neigungen bekämpfte. ${ }^{10}$ Georgs Nachfolger Joachim Friedrich, der von 1586 bis 1602 regierte, wandte sich von der strengen, kompromisslosen Durchsetzung der lutherischen Orthodoxie ab und praktizierte stattdessen einen Kurs der Toleranz gegenüber der reformierten Konfession auf Basis der gemässigt-ausgleichenden Theologie Melanchthons. Adam wurde also an einem Gymnasium unterrichtet, das in dieser Zeit sehr offen für calvinistische Positionen war, und dadurch auch in seiner persönlichen Glaubenshaltung geprägt. Unter diesen Vorzeichen lag für Adam natürlich der Wechsel in die reformierte Kurpfalz nahe.

Zwischen 1615 und 1620 gab Adam in Heidelberg 5 Bände mit insgesamt 546 Lebensläufen heraus. ${ }^{11}$ Dabei handelte es sich überwiegend um protestantische Gelehrte aus dem deutschsprachigen Kulturraum einschließlich angrenzender Gebiete wie der Schweiz und den Niederlanden; zeitlich deckt Adam die 150 Jahre ab etwa 1450 ab, wobei der Schwerpunkt mit zunehmender Personenzahl auf der zweiten Hälfte des 16. Jahrhunderts liegt. Er

10 Vgl. dazu Karl Friedrich Schönwalder, Geschichte des Königlichen Gymnasiums zu Brieg zur dreihundertjährigen Jubelfeier, Breslau 1869, S. 42-55.

11 Ich verwende in diesem Aufsatz die einbändige Gesamtausgabe: Melchior Adam, Dignorum Laude Virorum, Quos Musa vetat mori, Immortalitas, Seu Vitae Theologorum, Jure-Consultorum, et Politicorum, Medicorum atque Philosophorum, maximam partem Germanorum, Nonnullam quoque Exterorum, Editio tertia, Frankfurt am Main 1706. Diese umfasst fünf ursprünglich selbständig erschienene Teilbände mit jeweils eigener Paginierung: Vitae Germanorum Philosophorum, qui seculo superiori, et quod excurrit, philosophicis ac humanioribus literis clari floruerunt (1615); Vitae Theologorum exterorum principum, qui Ecclesiam Christi superiori seculo propagarunt et propugnarunt (1618); Vitae Germanorum Theologorum, qui superiori seculo ecclesiam Christi voce scriptisque propagarunt et propugnarunt (1620); Vitae Germanorum Iureconsultorum et Politicorum, qui superiori seculo, et quod excurrit, floruerunt (1620); Vitae Germanorum Medicorum, qui seculo superiori, et quod excurrit, claruerunt (1620). Vgl. zum biographischen Werk Adams Robert Seidel, Art. Adam, Melchior, in: Wilhelm Kühlmann (Hg.), Frühe Neuzeit in Deutschland 1520-1620. Literaturwissenschaftliches Verfasserlexikon, I, Berlin u.a. 2011, Sp. 26-31; Robert SEIDEL, Melchior Adams 'Vitae' (1615-1620) und die Tradition frühneuzeitlicher Gelehrtenbiographik: Fortschritte und Grenzen eines wissenschaftlichen Paradigmas um 1600, in: Gerhard Kosellek (Hg.), Oberschlesische Dichter und Gelehrte vom Humanismus bis zum Barock, Bielefeld 2000, S. 179-204; James Michael WeIss, The Harvest of German Humanism. Melchior Adam's Collective Biographies as Cultural History, in: Manfred P. Fleischer (Hg.), The Harvest of Humanism in Central Europe. Essays in Honor of Lewis W. Spitz, St. Loius 1992, S. 341-350; Dirk Werle, Melchior Adams Gelehrtenbiographien und ihr Bezug zur Enzyklopädistik, in: Martin Schierbaum (Hg.), Enzyklopädistik 1550-1650. Typen und Transformationen von Wissensspeichern und Medialisierungen des Wissens, Berlin - Münster 2009, S. 105-125. 
steht damit in der Tradition von Sammelbiographien und -bibliographien von Gelehrten, etwa Johannes Trithemius (1462-1516), Konrad Gesner (1516-1565), Heinrich Pantaleon (1522-1595) oder Nicolaus Reusner (1545-1602), die er auch als Quellen heranzieht. Adam selbst stellt sich - und daher auch der biographische Zugriff auf die Wissenschaftsgeschichte - in die antike Tradition exemplarischer Geschichtsschreibung: Sinn und Zweck der Viten sei es - so Adam in der Epistola dedicatoria der als erstes erschienenen Vitae Germanorum Philosophorum -, anhand der Lebensläufe die Leistungen und Tugenden der bedeutenden Deutschen der letzten 150 Jahre der Nachwelt exemplarisch vor Augen zu stellen. ${ }^{12}$

Adam strukturiert seine Sammlung entsprechend dem Aufbau der damaligen Universität und legt je einen Band mit Viten von Philosophen, Juristen und Medizinern und zwei Bände mit Theologenviten (deutsche und nicht-deutsche) vor. In der Hauptsache geht es um Universitätsprofessoren; insbesondere der Band zu den Jurisconsulti enthält allerdings auch Viten über Berater und Fürsten und Städten, wichtige städtische Ratsangehörige wie Pirckheimer und im weitesten Sinne "Politiker". Mit seinen Viten liefert Adam nicht nur einen Einblick in 150 Jahre Wissenschaftsgeschichte im deutschsprachigen Raum, sondern spiegelt auch wichtige Veränderungsprozesse im staatlichen und kirchlichen Bereich: Genannt sei nur die zunehmende Besetzung territorialstaatlicher Spitzenpositionen mit universitär qualifizierten Juristen, oft in Form einer Verbindung von Funktionen am Hof und an der Universität, oder die Organisation des reformatorischen Kirchenwesens mit der Verbindung von kirchlichen Führungspositionen und universitärem theologischen Lehramt - zwei Entwicklungsprozesse, die mein Aufsatz dann exemplarisch anhand der Viten Adams belegen wird.

Was Inhalte und Wertungen in seinen Viten betrifft, so ist Adam kein eigenständiger, kreativer Geist, sondern eher jemand, der vorhandene Quellen einfach reproduziert bzw. kompiliert. In vielen Fällen verwendet er eine einzige Quelle - oft den Personalia-Teil einer Leichenpredigt oder akademischen Gedenkrede für einen verstorbenen Universitätsprofessor, also einen eher panegyrisch geprägten Text -, in anderen kombiniert er mehrere Quellen. Als Quellen benutzt er eine Vielzahl historiographischer Gattungen: neben den bereits erwähnten Memorialtexten umfassende, oft wohlwollend-persönlich gefärbte Lebensbeschreibungen, sogenannte narrationes, Chroniken und andere historiographische Darstellungen, Briefsammlungen und manches mehr. Benutzt hat Adam dabei die reichen Bestände der Heidelberger Bibliotheca Palatina.

Adams Viten enthalten eine derartige Fülle an biographischen Details, dass man sie als eine für die Forschung wertvolle wissenschaftsgeschichtliche, sozial- und mentalitätsgeschichtliche Quelle betrachten kann: Die Lebensläufe gaben Auskunft über Geburt, Herkunft, Bildungsgänge, konfessionelle Positionierung, berufliche Positionen, Freundschaften, soziale Vernetzung, Heiraten und andere familiäre Bindungen des Betreffenden und vieles mehr. Anhand der Biographien lassen sich sehr gut und fundiert soziale Profile bestimmter Gruppen von Universitätsprofessoren der verschiedenen Fakultäten gewinnen und ihre Ausbildungs- und Karrierewege sowie die Profile ihres wissenschaftlichen Schaffens nachzeichnen.

12 Vgl. M. Adam, Vitae Germanorum Philosophorum, Epistola dedicatoria. 
Im Folgenden versuche ich nun, aus einigen ausgewählten Biographien Adams typische Ausbildungs- und Karrierewege sowie außeruniversitäre Tätigkeitsfelder von Professoren der theologischen und der juristischen Fakultät zu rekonstruieren. ${ }^{13}$ Adams Viten liefern viele biographische Fakten, die sich typologisch systematisieren lassen. Im Folgenden versuche ich, typische Karrieremuster von Professoren der Theologie und der Jurisprudenz aufzuzeigen. Insbesondere geht es darum, welche außeruniversitären Tätigkeitsfelder mit den Professorenstellen verknüpft waren, für welche Tätigkeitsfelder also Theologen und Juristen neben der Universität durch ihre Ausbildung qualifiziert waren. Dabei sind etwa 90 Prozent der biographischen Informationen aus Adam selbst gewonnen. ${ }^{14}$ Aufgrund der von mir gewählten Quellenbasis beschränke ich mich auf den protestantischen Bereich.

Beginnen wir mit den Theologen. Hier werde ich meinem Thema exemplarisch anhand von zwei Universitäten nachgehen. Dabei bietet es sich natürlich an, die Universität in den Vordergrund zu stellen, die für die Reformation in Deutschland impulsgebend war, nämlich Wittenberg. Charakteristisch für Wittenberger Theologieprofessoren in der frühen Phase der Reformation war die enge personelle und institutionelle Verflechtung mit kirchlichen, insbesondere kirchenleitenden Funktionen. Diese Verbindung von Professoren- und Pfarrstelle war gleichsam naturgegeben, denn in vorreformatorischer Zeit waren die Theologieprofessoren durch Pfründe des Wittenberger Allerheiligenstifts und damit gleichsam kirchlich finanziert, oder sie waren einem Orden zugeordnet wie z.B. die Professorenstelle des Augustinermönches Luther. Es gab ja noch keine rein universitär spezialisierte Professorenschaft, die Theologen für ebenso spezialisierte Kirchenstellen ausgebildet hat.

In anderen Fällen war es so, dass jemand, der andernorts mit der Lehre Luthers in Verbindung gekommen war, zunächst nach Wittenberg auf eine Pfarrstelle berufen wurde und erst später Professor an der theologischen Fakultät wurde - dies aber weiter in Verbindung mit einer kirchlichen Funktionsstelle. Nach den Kirchenvisitationen 1528/1529 entstanden dann ab den 1530er Jahren in Kursachsen erste Anfänge eines vom Kurfürsten geleiteten hierarchisch strukturierten Kirchenwesens mit Superintendenten und Generalsuperintendenten für die Dienstaufsicht über die Gemeinden sowie Konsistorien für Kirchenverwaltung, Kirchenjustiz und Ehegerichtsbarkeit. Für diese Funktionsstellen wurden dann Theologieprofessoren mit einem speziellen Qualifikationsmerkmal, der Promotion, herangezogen. Letztere war ja durchaus noch nicht allgemeine Eingangsvoraussetzung für theologische Professuren. ${ }^{15}$

13 Die Mediziner bleiben aus Zeit- und Raumgründen ausgespart. Vgl. exemplarisch zu Studium und nachuniversitären Karrieren Kölner Mediziner im Spätmittelalter und beginnendem 16. Jahrhundert Markus Bernhardt, Gelehrte Mediziner des späten Mittelalters: Köln 1388-1520. Zugang und Studium, in: Rainer Christoph Schwinges (Hg.), Gelehrte im Reich. Zur Sozial- und Wirkungsgeschichte akademischer Eliten des 14. bis 16. Jahrhunderts, Berlin 1996, S. 113-134, sowie Cay-Rüdiger PrüLL, Die „, Karriere “ der Heilkundigen an der Kölner Universität zwischen 1389 und 1520, ibidem, S. 135-158.

14 Die exakten Jahreszahlen sind im Folgenden allerdings oft aus modernen biographischen Lexika oder der Sekundärliteratur ergänzt. Bei Adam finden sich oft Angaben wie ,vicesimum aetatis annum ingressus“ o.ä. Da es mir nicht um die Erörterung wissenschaftlicher Detailprobleme zu den einzelnen exemplarisch vorgestellten Personen, sondern lediglich um einen systematisierenden Überblick über typische Karriereverläufe anhand biographischer Daten geht, verzichte ich bei den von mir ausführlicher dargestellten Personen auf umfangreichere Literaturangaben. Die von mir vergleichend und zur Ergänzung des von Adam gelieferten Datenmaterials herangezogenen Lexika oder biographischen Darstellungen gebe ich jeweils an Ort und Stelle an. Artikel aus der ADB werden nur nachgewiesen, wenn keine neueren Lexikonartikel zur Verfügung stehen.

15 Noch bei der Berufung Erhard Schnepfs als Professor für Theologie mit dem Schwerpunkt Hebräisch an die Universität Tübingen im Februar 1544 bemängelte der Senat der Universität Schnepfs fehlende theologische 
Insgesamt lassen sich - bei allen Unterschieden im Detail - aus den Viten früher Wittenberger Theologieprofessoren einige typische Strukturmerkmale herauspräparieren: Erste kirchliche oder akademische Sozialisation im vorreformatorischen Umfeld - Berührung mit der reformatorischen Bewegung über Kontakte oder die Schriften Luthers - z.T. Wechsel nach Wittenberg und dort neben dem Artesstudium Studium der Theologie - relativ schnelle Übernahme kirchlicher Ämter - Erwerb eines höherqualifizierenden Abschlusses in Form der theologischen Doktorpromotion - Übernahme höherrangiger kirchlicher Ämter sowie theologischer Professuren aufgrund Bestallung durch den Landesherrn - externe Tätigkeit als Kirchenreformer und Kirchenorganisator.

Gerade der letztgenannte Punkt berührt - neben der kirchenleitenden Funktionen in, wenn man so will, der eigenen kursächsischen Landeskirche - eine weiteres Tätigkeitsfeld der frühen Wittenberger Theologieprofessoren: Wittenberger waren eingebunden in die obrigkeitlich organisierte territoriale und städtische Reformation über Kursachsen hinaus, sie wurden andernorts zu Reformatoren.

Diese Sachverhalte sollen nun an zwei Beispielen illustriert werden: Johannes Bugenhagen (1485-1558), ${ }^{16}$ in Wollin im Herzogtum Pommern geboren, nahm er nach kurzem Artesstudium in Greifswald eine Schulstelle im pommerschen Treptow an. Ab 1517 war er Lektor für Bibelexegese und Theologie an der pommerschen Ordensschule der Prämonstratenser. Durch die Beschäftigung mit Luthers Schriften lernte Bugenhagen dann die Grundzüge des lutherisch-reformatorischen Glaubensverständnisses kennen. 1521 immatrikulierte sich Bugenhagen in Wittenberg und hielt von Anfang an exegetische Vorlesungen an der Universität. Im Oktober 1523 wurde er Pfarrer an der Stadtkirche in Wittenberg.

Auf dieser Grundlage avancierte Bugenhagen nun zum Organisator des reformatorischen Kirchenwesens an zahlreichen Orten außerhalb Kursachsens: 1528 in der Stadt Braunschweig, 1528/1529 in Hamburg, 1530 bis 1532 in Lübeck. Zu diesen Einsätzen wurde er jeweils in Wittenberg angefordert. Dann schuf er dort in Form der Kirchenordnungen die organisatorischen Grundlagen des reformatorischen Kirchenwesens, kümmerte sich um Gottesdienst, Armenfürsorge, Diakonie und Schulwesen, besetzte die wichtigsten Positionen, v.a. die Superintendentenstellen, und kehrte jeweils nach Wittenberg zurück.

Interessant sind nun die Hintergründe von Bugenhagens theologischer Promotion im Juni 1533. Bugenhagen hatte es bisher universitär nur zum Lizentiaten der Theologie gebracht und stand der Promotion zunächst sehr unwillig gegenüber. Unterstützt wurde die Fakultät

Promotion. Schnepf verwies darauf, dass er sich bereits im Studium in Heidelberg nach der Promotion zum Magister Artium fünf Jahre in der Theologie auf die Doktorpromotion vorbereitet, alle erforderlichen Disputationen und Prüfungen absolviert und in der Theologie den Grad des Baccalaureus erreicht hat. Zudem könne er wegen seiner vielen Kinder nicht die hohen Kosten für eine theologische Doktorpromotion aufbringen. Dennoch beschlossen der Rektor und die Ordinarien der Universität, Schnepf im Februar 1544 ihren Statuten gemäß zu promovieren. Vgl. Hermann EhmER, Erhard Schnepf - ein Lebensbild, Blätter für Württembergische Kirchengeschichte 87, 1987, S. 72-126, hier S. 104-107.

16 Vgl. M. Adam, Vitae Germanorum Theologorum, S. 150-154; Friedrich Wilhelm Bautz, Art. Bugenhagen, Johannes, BBKL 1/21990, S. 805-807; Norbert Buske, Johannes Bugenhagen. Sein Leben - Seine Zeit - Seine Wirkungen, Schwerin 2010; Volker Gummelt, Bugenhagens Tätigkeit an der Universität Wittenberg, ZKG 105/1994, S. 191-201; Volker Gummelt, Johannes Bugenhagen, in: Irene Dingel - Volker Leppin (Hgg.), Reformatorenlexikon, Darmstadt 2014, S. 74-81; Hans Hermann Holfelder, Art. Bugenhagen, Johannes, TRE 7/1981, S. 354-363; Thomas Kaufmann, Art. Bugenhagen, Johannes, DBETh 1/2005, S. 204 f.; Gerhard MüLler, Art. Bugenhagen, Johannes, RGG4 1/1998, S. 1852-1854; Ernst Wolf, Art. Bugenhagen, Johannes, NDB 3/1957, S. 9 f. 
in ihrem Ansinnen massiv von Kurfürst Johann Friedrich, der den bisherigen Wittenberger Stadtpfarrer für eine Superintendentenstelle vorgesehen hatte. Hier wird der über die Universität hinausweisende Aspekt der theologischen Promotion erkennbar: Sie sollte zum Qualifikationsmerkmal für evangelische Theologen werden, mit denen leitende Kirchenstellen besetzt wurden.

Bugenhagens weitere Karriere ist dann geprägt vom Nebeneinander von Universitätstätigkeit, kirchenleitenden Funktionen im Kurfürstentum und weiteren kirchenreformatorischen bzw. -organisatorischen Einsätzen auswärts: Bugenhagen wurde Obersuperintendent der rechtselbischen Gebiete des Kurfürstentums. Seit 1535 war er einer der vier ordentlichen Professoren der Wittenberger theologischen Fakultät; diese Professur war fortan mit dem Wittenberger Stadtpfarramt und der Superintendentur verbunden. Außerhalb Kursachsens war er entscheidend an der Kirchenreform im Herzogtum Pommern auf Einladung der dortige Herzöge 1534 beteiligt, ebenso an den Kirchenreformen im Herzogtum BraunschweigWolfenbüttel, in den Herzogtümern Schleswig und Holstein, in Hildesheim, in Norwegen und in Dänemark. Alle diese Tätigkeiten vollzog er unter Beibehaltung seines Wittenberger Doppel- oder Dreifachamtes als Pastor, Superintendent und Theologieprofessor.

Justus Jonas (1493-1555), ${ }^{17}$ geboren in Nordhausen, war ab Anfang 1519 Professor in der juristischen Fakultät in Erfurt. Nach ersten Kontakten mit der reformatorischen Bewegung wechselte er Mitte 1520 nach Wittenberg und wurde Propst des Wittenberger Allerheiligenstiftes, eine Kirchenstelle, die traditionell mit einer Professur für kanonisches Recht in der juristischen Fakultät verbunden war. Jonas vollzog nun aber den Wechsel in die theologische Fakultät und wurde dort zum Doktor der Theologie promoviert. Die Einkünfte der Stiftspropstei, die Jonas behielt, waren damit gleichsam der juristischen Fakultät entzogen und zugunsten einer reformatorisch-theologischen Professorenstelle umgewidmet worden. 1525 wurde das Stift dann ganz vom Kurfürsten eingezogen und seine Einkünfte zur Finanzierung der Wittenberger Professorenstellen aus dem kurfürstlichen Besoldungsfond verwendet.

Später begegnet Jonas dann als Organisator und Durchführer von Kirchenvisitationen in Kursachsen 1528 bis 1530, als Pfarrer der Wittenberger Schlosskirche sowie mehrmals als Dekan der Fakultät und Rektor der Universität. Nach 1530 entwickelte er zumindest als Mitbeteiligter Kirchenordnungen, vor allem für das bis 1537 katholische Herzogtum Sachsen. Die kirchenpolitische Zusammenarbeit unter den reformatorischen Fürsten belegt sehr schön der Hinweis bei Adam, dem evangelisch gewordenen Herzog Heinrich seien von Kurfürst Johann Friedrich Georg Spalatin, Nikolaus Amsdorf und eben Justus Jonas als Kirchenvisitatoren geschickt worden.

Wie intensiv die überterritoriale Vernetzung der reformatorischen Bewegung und der entsprechende Austausch geeigneten Personals inzwischen war, zeigt Jonas' Berufung nach Halle im Jahre 1541, die zugleich eine Aufgabe seiner Wittenberger Funktionen bedeutete.

17 Vgl. M. Adam, Vitae Germanorum Theologorum, S. 125 f.; Walter Delius, Leben und Lehre. Justus Jonas 1493-1555, Gütersloh 1952; Ders., Art. Jonas, Justus, NDB 10/1974, S. 593 f.; Irene Dingel (Hg.), Justus Jonas (1493-1555) und seine Bedeutung für die Wittenberger Reformation, Leucorea-Studien zur Geschichte der Reformation und der Lutherischen Orthodoxie, XI, Leipzig 2009; Karin Grol, Art. Jonas, Justus, BBKL 3/21992, S. 636 f.; Hans-Günter Leder, Art. Jonas, Justus, TRE 7/1981, S. 234-238; Inge Mager, Das war viel ein anderer Mann. Justus Jonas, ein Leben mit und für Luther, in: Peter Freybe (Hg.), Luther und seine Freunde, Wittenberg 1998, S. 10-27; Bernd MoELLER - Bruno JAHN, Art. Jonas, Justus, DBETh 1/2005, S. 734; Helmut Zsсносн, Art. Jonas, Justus, RGG44 4/2001, S. 569 f. 
Halle war seinerzeit Residenzstadt des Erzbischofs von Magdeburg; dieser hatte, wie Adam schreibt, die öffentliche Ausübung der wahren Religion erlaubt, Klöster und Kanonikerkollegien aber in altem Zustand belassen. Der Stadtrat wollte nun die städtischen Pfarreien mit evangelischen Predigern besetzen und fragte beim sächsischen Kurfürsten an; dieser entsandte Jonas, der dann dort die neue evangelische Kirchenordnung ausarbeitete und später Superintendent wurde. In einer späteren Phase seines Lebens kehrte Jonas dann noch einmal in das ernestinesche Sachsen zurück und wurde Hofprediger und Superintendent in Coburg.

Die überterritoriale Verwendung von Theologieprofessoren in kirchenreformatorischer oder kirchenleitender Funktion blieb für die erste Hälfte des 16. Jahrhundert ebenso kennzeichnend wie der prägende Einfluss Wittenbergs. Impulsgebend waren in vielen Fällen Luther und Melanchthon, die ihre besten Studenten oder Kollegen für solche Positionen in anderen Territorien empfahlen. Ausgehandelt wurde der Wechsel dann, soweit es um fürstlich oder städtisch bestallte Professoren ging, zwischen den jeweiligen Landesherren oder städtischen Obrigkeiten.

Machen wir nun einen Sprung in die Mitte des 16. Jahrhunderts an eine Universität der ersten nachreformatorischen Generation, nämlich nach Rostock, die Universität des Herzogtums Mecklenburg. ${ }^{18}$ Dort hatte sich die Reformation ab den 1530er Jahren schrittweise, aber in einzelnen Landesteilen mit unterschiedlicher Geschwindigkeit durchgesetzt. 1549 lehnte der Mecklenburgische Landtag das Augsburger Interim ab und verabschiedete eine eigene mecklenburgische Konfession auf Basis der Confessio Augustana. Damit galt für das gesamte Territorium, d.h. die Herrschaftsgebiete beider Teillinien der mecklenburgischen Herzöge, die Reformation als eingeführt. An der 1552 erlassenen Kirchenordnung waren nun vor allem Rostocker Theologieprofessoren maßgeblich beteiligt.

Grundsätzlich lassen sich, vor dem Blick auf einige Einzelbeispiele, vier Dinge zu den Rostocker Theologieprofessoren sagen: 1. Die Professoren der ersten Generation, also der 1550er Jahre, waren in aller Regel in Wittenberg ausgebildet worden, hatten dort promoviert und z.T. erste Lehrerfahrungen in der dortigen Artistenfakultät oder in der theologischen Fakultät gesammelt. Später trat der Wittenberger Einfluss zurück, die Professoren hatten dann eher in Jena, Leipzig, Greifswald, Helmstedt oder Rostock selbst studiert und in Rostock promoviert. 2. Die Statuten der Fakultät machten konkrete Vorgaben, was das Anforderungsprofil der Professoren betrifft: Diese mussten den theologischen Doktorgrad erworben haben und gemäß dem in Mecklenburg geltenden Bekenntnis lehren. 3. Die Professoren wurden von der jeweiligen berufenden Instanz in festen kirchlichen Ämtern eingesetzt oder zumindest für kirchenpolitische Aufgaben herangezogen. 4. Die ab 1560 berufenen Professoren blieben im allgemeinen für den Rest ihres Lebens im Herzogtum, das heißt: Wir haben es in Mecklenburg mit dauerhaften, sich personell verstetigenden Strukturen zu tun, und reformatorische Impulse, die von Mecklenburg aus auf andere Territorien ausstrahlten, gingen immer von einem Amtsträger aus, der institutionell in Mecklenburg verwurzelt war und blieb.

18 Theologie und Theologieprofessoren an der Universität Rostock im Zeitalter der Konfessionalisierung, also ab Mitte des 16. Jahrhunderts, sind besonders gut erforscht aufgrund der Studie von T. KAUFMANN, Universität und lutherische Konfessionalisierung, hier zu „Kollektivbiographie“ der Professoren und zu ihrer Einbindung in außeruniversitäre, v.a. kirchliche institutionelle Kontexte, S. 131-232. Kaufmann arbeitet v.a. auf das Basis von Leichenpredigten als Quellmaterial. 
Wenn eben bei Punkt drei von den berufenden Instanzen die Rede war, so bedeutet das, dass die Rostocker Theologieprofessoren z.T. vom Rat der Stadt Rostock, z.T. durch die Herzöge berufen und besoldet wurden; es gab also „städtische“ und „,ürstliche“ Professoren. Die zwei städtischen Professoren waren zugleich Pfarrer in Rostock an einer der vier Rostocker Gemeinden; der aus dem Kreis der Rostocker Pfarrer gewählte und vom Rat eingesetzte Rostocker Stadtsuperintendent war in der Regel zugleich Professor an der Universität.

Für den Aufbau des evangelischen Kirchenwesens im gesamten Territorium, die Ausgestaltung der Kirchenordnung sowie für kirchenleitende Funktionen zogen die Herzöge dann ,ihre“ Professoren heran: Diese waren beteiligt an Visitationen, berieten die Fürsten in kirchlichen Angelegenheiten, waren an der Kirchenordnung von 1552 - die übrigens von einem Rostocker Professor, Johannes Aurifaber (1517-1568), in direkter Zusammenarbeit mit Melanchthon verfasst worden war - und ihren Revisionen beteiligt, wirkten an der Konsistorial- und Superintendentenordnung mit und wurden von den Herzögen als Superintendenten an die Spitze der verschiedenen mecklenburgischen Kirchenbezirke berufen. In dieser Funktion oblag den Professoren zusammen mit den jeweiligen städtischen Schulträgern die Aufsicht über die Schulen ihres Bezirks, die Prüfung und Ordination der Pfarrkandidaten, die Visitation der Gemeinden und die Lehraufsicht über die Pfarrer. Schließlich waren manche Professoren Mitglied der obersten kirchenleitenden Behörde, des Konsistoriums, und kümmerten sich dort um Lehrstreitigkeiten, moralische Vergehen von Pfarrern, Exkommunikationsverfahren, um die Ehegerichtsbarkeit und das Schulwesen.

Einige der genannten Aspekte lassen sich anhand der Vita von David Chytraeus aufzeigen (1530-1600). ${ }^{19}$ Der in der Grafschaft Hohenlohe als Sohn eines Pastors geborene Chytraeus hatte zunächst in Tübingen Artes und Theologie studiert, war 1544 dann als Magister nach Wittenberg gegangen, hatte dort sein Theologiestudium fortgesetzt und als Magister Privatvorlesungen über Melanchthons Loci und Rhetorik gehalten. Melanchthon, in dessen Hausgemeinschaft er aufgenommen worden war, wurde sein Lehrer und großes Vorbild. Über seine Berufung nach Rostock im Jahre 1550, zunächst als Dozent an das Pädagogium der Universität, berichtet Adam, Arnold Burenius, ein Rostocker Artistenmagister, sei im Auftrag des Herzogs nach Wittenberg gekommen und habe mit Melanchthon, verhandelt,

19 Vgl. M. Adam, Vitae Germanorum Theologorum, S. 323-330; Peter F. Barton, Art. Chyträus, David, TRE 8/1981,S. 88-90; Friedrich Wilhelm BAuTz, Art. Chytraeus (gräzisiert aus Kochhafe), David, BBKL 1/21990, S. 1021 f.; Harald BollBucK, Geschichts- und Raummodelle bei Albert Krantz (um 1448-1517) und David Chytraeus (1530-1600). Transformationen des historischen Diskurses im 16. Jahrhundert, Frankfurt a.M. Berlin - Bern - Brüssel - New York - Oxford - Wien 2006, S. 151-169; Otfried CzaikA, Art. Chytraeus, David, Frühe Neuzeit in Deutschland. Literaturwissenschaftliches Verfasserlexikon 1/2011, S. 511-521; Thomas Fuchs, David und Nathan Chytraeus. Eine biographische Annäherung, in: Karl-Heinz Glaser - Hanno Lietz Stefan Rhein (Hgg.), David und Nathan Chytraeus. Humanismus im konfessionellen Zeitalter, Ubstadt-Weiher 1993, S. 33-48; Thomas Kaufmann, Die Brüder David und Nathan Chytraeus in Rostock, ibidem, S. 103-116; Ders., Universität und lutherische Konfessionalisierung, Kapitel II, Die Rostocker Professoren - Personen und Ämter, S. 129-250; Ders., Art. Chyträus, David, RGG4 2/1999, S. 377 f.; Rudolf Keller, Der Beitrag des David Chytraeus zur Einigung des Luthertums, in: K.-H. Glaser - H. Lietz - S. Rhein (Hgg.), David und Nathan Chytraeus, S. 117-128; Ders., David Chytraeus (1530-1600). Melanchthons Geist im Luthertum, in: Heinz Scheible (Hg.), Melanchthon in seinen Schülern, Wiesbaden 1997, S. 361-371; Bernd MoELLER - Bruno JAHN, Art. Chytraeus, David, DBETh 1/2005, S. 237 f.; Steffen Stuth, David Chytraeus. Ein Gelehrter der Universität Rostock im Umkreis der mecklenburgischen Landesfürsten, in: Bernd Röcker (Hg.), Reformation und Humanismus im Kraichgau, Eppingen 2003, S. 117-125; Ernst Wolf, Art. Chytraeus, David, NDB 3/1957, S. 254. 
wen er für eine theologische Professur in Rostock empfehlen könne. Melanchthon nannte Johannes Aurifaber, der in Wittenberg eine ordentliche Professur für Mathematik innehatte, und eben Chytraeus. Beide wechselten nach Rostock: Aurifaber als Professor für Theologie und Pastor in Rostock, Chytraeus als Dozent für Griechisch und Latein, Philosophie, Geschichte und Theologie am Pädagogium. Nach seiner Promotion 1561 wurde Chyträus 1563 vom Herzog auf eine ordentliche Professur für Theologie an der Universität berufen. Dabei hielt er weiter Vorlesungen zu antiken Historikern in der Philosophischen Fakultät: ein gleichzeitiges Wirken in mehreren Fakultäten, wie wir es schon von Melanchthon her kennen und damit ebenfalls ein typisches Merkmal für Professoren des 16. Jahrhunderts.

Bemerkenswert bei Chytraeus ist nun, dass er zeitlebens kein pastorales Amt ausübte, aber dennoch im kirchlichen Bereich umfassend wirksam war. Zum einen im Herzogtum selbst: er wirkte an Visitationen, an der mecklenburgischen Konsistorialordnung und Superintendentenordnung mit, 1578 und 1589 war er maßgeblich an Überarbeitungen der Kirchenordnung beteiligt. Ab 1570 war er Mitglied des mecklenburgischen Konsistoriums. Über den ganzen Zeitraum war er theologischer Berater der mecklenburgischen Herzöge.

Zum zweiten wirkte er als Kirchenorganisator über die Grenzen Mecklenburgs hinaus: Auf Veranlassung Kaiser Maximilians II. verfasste Chytraeus für die evangelischen Stände Niederösterreichs und der Steiermark Kirchenordnungen. Dass prominente und profilierte Experten über die Grenzen des eigenen Territoriums und ihres eigentlichen universitären Aufgabenbereiches hinaus wirksam werden konnten, zeigt sich bei Chytraeus an zwei weiteren Beispielen: Von Herzog Julius von Braunschweig wurde Chytraeus 1575 als Berater für die Gründung und den Aufbau der Universität Helmstedt herangezogen - für diese Aufgabe wurde Chytraeus vom mecklenburgischen Herzog richtiggehend für ein Jahr ausgeliehen. Im Anschluss daran wurde Chytraeus von Kurfürst August von Sachsen für die abschließenden Beratungen über die Konkordienformel engagiert - dies alles auf Basis einer Professorenstelle in Rostock, der Chytraeus trotz aller Abwerbeversuche von anderen Universitäten treu blieb.

Typisch für die Verbindung von Professorenstelle und kirchlichem Amt ist die Karriere von Lucas Bacmeister (1530-1608), ${ }^{20}$ der nach Theologiestudium in Wittenberg und einer Hofpredigertätigkeit in Dänemark 1562 nach Rostock kam. Dort wurde er vom Rat als Pfarrer der Marienkirche und Professor für Theologie berufen und wurde sofort zum Superintendenten gewählt. Die für die Professorenstelle dringend nötige theologische Promotion holte er zwei Jahre später nach. Auch er wirkte dann im Gefolge von Chytraeus als Visitator der Kirchen und Schulen in Österreich, wofür er, wie Adam schreibt, die Genehmigung des mecklenburgischen Herzogs, des Rats der Universität und des Stadtrates von Rostock benötigt - ein gutes Beispiel dafür, welche Entscheidungsträger bei universitären Personalfragen beteiligt waren. Und auch Bacmeister vermochten Geld und Ehre (,omnes tam honorificae tam opimae conditiones" ${ }^{21}$ anderer Angebote nicht aus Rostock wegzulocken.

Die Professoren der Jurisprudenz im 16. Jahrhundert gehörten zu den universitär ausgebildeten territorialstaatlichen Eliten und nahmen Spitzenfunktionen ganz unterschiedlicher Art wahr: Sie waren als fürstliche Räte Mitglieder des obersten Gerichts bzw. Hofgerichts,

20 Vgl. M. Adam, Vitae Germanorum Theologorum, S. 372-379; Friedrich Wilhelm BaUtZ, Art. Bacmeister, Lucas der Ältere, BBKL 1/21990, S. 329; Bernd Moeller - Bruno JAHN, Art. Bacmeister, Lucas d.Ä., DBETh 1/2005, S. 77.

21 M. ADAM, Vitae Germanorum Theologorum, S. 378. 
saßen neben den führenden Theologen in den kirchenleitenden Konsistorien bzw. Kirchenräten, bildeten als Kanzler, Räte oder Rechtsberater unter dem Fürsten das höchste Gremium der Landesregierung und wurden insbesondere als Gesandte für diplomatische Missionen eingesetzt. In aller Regel entstammten sie, zumindest was die bei Adam dokumentierten Viten betrifft, nicht dem Adel, sondern dem städtischen Bürgertum. ${ }^{22}$

Allerdings lässt sich dieser Zusammenhang, zumindest für das frühe fünfzehnte Jahrhundert, nicht umgekehrt formulieren: Nicht alle leitenden fürstlichen Beamten waren auch Juraprofessoren, manche nicht einmal studierte Juristen. So hatte Gregor Brück (14831557), ${ }^{23}$ geboren in einer Kleinstadt bei Wittenberg als Sohn des dortigen Bürgermeisters, in Frankfurt/Oder und Wittenberg Jurisprudenz studiert und sogar 1509 promoviert, bevor er als Kanzler an den Hof des sächsischen Kurfürsten Friedrich des Weisen berufen wurde. Diesem und seinen Nachfolgern diente er dann bei der Organisation des reformatorischen Kirchenwesens sowie als Gesandter auf diversen Reichstagen. Eine juristische Professur in Kursachsen bekleidete er nicht.

Juraprofessoren scheinen zunächst vor allem dann herangezogen worden zu sein, wenn eine Stelle im obersten territorialen Gericht zu besetzen war. So hatte Hieronymus Schurff (1481-1554), ${ }^{24}$ als Arztsohn in St. Gallen geboren, in Wittenberg Jurisprudenz studiert und war dort auch promoviert worden. Ab 1507 war er dort Professor für Römisches Recht und wurde dann als kursächsischer Rat Richter am kursächsischen Oberhofgericht. Interessant in diesem Zusammenhang ist eine Bemerkung Adams, die Richter am Oberhofgericht seien „ex Nobilitate \& ex Doctoribus“ 25 berufen worden - ein Zeichen für das Nebeneinander von traditioneller Rekrutierung aus dem Adel und zunehmender Professionalisierung mit Zugriff auf wissenschaftlich ausgebildete Vertreter des städtischen Bürgertums in den territorialstaatlichen Spitzenpositionen der Zeit - Leute, für die das Studium also die Möglichkeit zum sozialen Aufstieg eröffnete. Als Schurff nach dem Schmalkaldischen Krieg dann nach Brandenburg auf eine juristische Professur in Frankfurt/Oder gewechselt war,

22 Zur Bedeutung universitär ausgebildeter Juristen für die Territorialstaatsbildung vgl. den grundlegenden Aufsatz von Notker Hammerstein, Universitäten - Territorialstaaten - Gelehrte Räte, in: Notker Hammerstein, Res publica litteraria. Ausgewählte Aufsätze zur frühneuzeitlichen Bildungs-, Wissenschaftsund Universitätsgeschichte, hg. v. Ulrich Muhlack und Gerrit Walther, Berlin 2000, S. 257-302, mit einem Vergleich zu den von mir hier nicht berücksichtigten katholischen Territorien. Vgl. Rainer A. MüLLER, Zur Akademisierung des Hofrats. Beamtenkarrieren im Herzogtum Bayern 1450-1650, in: R. Ch. Schwinges (Hg.), Gelehrte im Reich, S. 291-307. Zu den spätmittelalterlichen Wurzeln der Verwendung von Juristen im Dienst von Territorialherren vgl. Ingrid MännL, Die gelehrten Juristen im Dienst der Territorialherren, ibidem, S. 269-290.

23 Vgl. M. Adam, Vitae Germanorum Jureconsultorum et Politicorum, S. 51-53; Friedrich Wilhelm Bautz, Art. Brück (eigentlich: Heinse; latinisiert: Portanus [ita]), Gregor, BBKL 1/21990, S. 762 f.; Ulrich v. BRÜcK, Im Dienste der Reformation. Ein Lebensbild des kursächsischen Kanzlers Gregor von Brück, Berlin 1985; Ekkehart FaBian, Art. Brück, Gregor, NDB 2/1955, S. 653 f.; DeRs., Dr. Gregor Brück 1557-1957: Lebensbild und Schriftwechselverzeichnis des Reformationskanzlers, Tübingen 1957; DeRs., Art. Brück, Gregor, TRE 7/1981, S. 212-216; Bernd Moeller - Bruno JaHn, Art. Brück, Gregor, DBETh 1/2005, S. 187; Heinz SCheIBLE, Art. Brück, Gregor, RGG4 1/1998, S. 1778.

24 Vgl. M. AdAm, Vitae Germanorum Jureconsultorum et Politicorum, S. 44-46; Heiner LÜCK, ... und viell feiner gesellen, die fleißiglich studieren ...: Hieronymus Schurff (1481-1554), in: Peter Freybe (Hg.), Wittenberger Lebensläufe im Umbruch der Reformation, Wittenberg 2005, S. 52-74; Wiebke SснаICh-KLose, D. Hieronymus Schürpf. Leben und Werk des Wittenberger Reformationsjuristen 1481-1554, Trogen (Schweiz) 1967; Rolf STEDING, Hieronymus Schürpf und sein Verhältnis zu Martin Luther. Porträtskizze eines nahmhaften Wittenberger Juristen, in: Jus Commune. Zeitschrift für europäische Rechtsgeschichte 20, 1993, S. 186-192; Andreas Otto WeBer, Art. Schurff, Hieronymus, NDB 23/2007, S. 760 f.

25 M. AdAm, Vitae Germanorum Jureconsultorum et Politicorum, S. 45. 
bot ihm Kaiser Karl V. eine Berufung an das Reichskammergericht an, was Schurff jedoch aus Altersgründen ablehnte.

Die Verknüpfung mit wichtigen Hofämtern häuft sich bei den in der Mitte des Jahrhunderts ins Professorenamt gekommenen Juristen. In manchen Fällen verbrachten die Professoren dabei ihr gesamtes Berufsleben im gleichen Territorium: Dies gilt für Nikolaus Varnbüler (1519-1604), ${ }^{26}$ geboren als Sohn eines Lindauer Bürgermeisters, von 1554 bis 1594 Professor für Römisches Recht in Tübingen und in dieser Zeit Herzoglicher Rat, Mitglied des Hofgerichts und Gesandter des Württembergisches Herzogs auf diversen Reichstagen. Ähnlich Johannes Schneidewein (1519-1568), ${ }^{27}$ Sohn eines Beamten der Grafen von Stolberg: Nach Studium der Jurisprudenz in Wittenberg erhielt er dort 1551 die Professur für Römisches Recht, promovierte zwei Jahre später und wurde dann Mitglied im Hofgericht des Kurfürstentums sowie im Rat der kurfürstlichen Rechtsberater, schließlich Vertreter des Kurfürstentums am Reichskammergericht. Daneben war er als Diplomat im Dienst der Kurfürsten aktiv.

Bei anderen wiederum ist ein Wechsel der Universität über die Grenzen des Territorialstaats hinaus zu verzeichnen. An zwei Universitäten in zwei Territorien, nämlich Jena und Wittenberg, war Matthias Wesenbeck (1531-1586), ${ }^{28}$ geboren als Sohn eines Antwerpener Stadtrats, tätig, und auch seine Promotion erfolgte erst nach Beginn der Vorlesungstätigkeit in Jena. Während seiner elfjährigen Tätigkeit in Jena im Herzogtum Sachsen ab 1558 nahm er Aufgaben am Hof wahr;; ${ }^{29}$ während seiner Lehrtätigkeit in Wittenberg im Kurfürstentum Sachsen, die er weitere siebzehn Jahre bis zu seinem Tod 1586 ausübte, wirkte er in der Funktion eines Geheim- oder Appellationsrates des Kurfürsten als Richter und Anwalt am Hofgericht.

Belegt ist auch der Wechsel von einer Professorenstelle auf eine Hofstelle in einem anderen Territorium, wobei Adam häufig die Niederlage der Protestanten im Schmalkaldischen Krieg als Grund angibt. So war Johannes Lucanus (gest. 1562) ${ }^{30}$ ab 1543 Professor für Jurisprudenz in Wittenberg und wurde dann 1547 von Herzog Albrecht von Mecklenburg als Kanzler berufen. Dort kümmerte er sich um den Wiederaufbau der evangelischen Kirche nach dem Interim, um das Schul- und Gerichtswesen und die Universität Rostock.

Auffällig ist, dass einige der Genannten auch Aufgaben außerhalb ihres Territorium wahrnahmen: So wirkte der Württemberger Varnbüler zugleich als juristischer Berater des Markgrafen von Brandenburg und des Herzogs von Preußen sowie als deren Vertreter vor dem Reichskammergericht. Auch die Kontakte von Lucanus zu seinem späteren Arbeitgeber nach Mecklenburg waren bereits in seiner Zeit als Professor in Wittenberg geknüpft worden: Einige Male, so schreibt Adam, sei er von den Mecklenburgischen nobiles als Berater in Rechtsangelegenheiten herangezogen worden. ${ }^{31}$ Michael Teuber schließlich, der

26 Vgl. M. Adam, Vitae Germanorum Jureconsultorum et Politicorum, S. 181-186; Friedrich WintterLin, Art. Varnbüler, Nikolaus, ADB 39/1895, S. 498 f.

27 Vgl. M. AdAm, Vitae Germanorum Jureconsultorum et Politicorum, S. 81-84; v. JACOBI, Art. Schneidewein, Heinrich und Johannes, ADB 32/1891, S. 144-149.

28 Vgl. M. Adam, Vitae Germanorum Jureconsultorum et Politicorum, S. 123-126.

29 Adam spricht davon, dass er ,auch beim Zusammentreffen gewichtigster Aufgaben, wie sie der Hof aufzubürden pflegt, niemals, außer in äußerster Zwangslage, seine Lehrtätigkeit zu unterbrechen pflegte."Vgl. M. Adam, Vitae Germanorum Jureconsultorum et Politicorum, S. 124.

30 Vgl. M. Adam, Vitae Germanorum Jureconsultorum et Politicorum, S. 70-72.

31 „Aliquoties enim e Wittembergensi Academia a nobilibus eo venerat, qui consilio eius \& opera in iudiciis \& causis usi.“ M. AdAm, Vitae Germanorum Jureconsultorum et Politicorum, S. 71. 
ja bereits in Kursachsen Erfahrungen in der Ehegerichtsbarkeit gesammelt hatte, wirkte während seiner Zeit als Kanzler des Bistums Cammin am Aufbau der Matrimonialgerichtsbarkeit im Herzogtum Pommern-Stettin mit.

Von den Genannten hatten Brück, Schneidewein und Lucanus Artes- und Jurastudium dort verbracht, wo sie auch später angestellt wurden: Ein Beleg für die zunehmende Territorialisierung und Provinzialisierung der Universitäten, die vorrangig für den Bedarf des eigenen Territoriums ausbildeten. Die Fürsten versuchten dann natürlich, diese Fachkräfte im Land zu halten. Andererseits geben die Biographien an vielen Stellen zu erkennen, wie sich Fürsten zumindest innerhalb der gemeinsamen Konfession über die Landesgrenzen hinweg mit Personal aushalfen, aber auch wissenschaftlich qualifiziertes Personal abwarben.

Die juristische Doktorpromotion war inzwischen - wenn auch nicht immer als Eingangsvoraussetzung - für die Professorentätigkeit obligatorisch geworden. Sie scheint damit auch für die mit den Professorenstellen verbundenen exponierten Positionen der Standard geworden zu sein. Diese Qualifikationen wurden in der Mitte des Jahrhunderts zunehmend an den juristischen Fakultäten deutscher Universitäten erworben. Daneben gab es aber weiter den zu Beginn des Jahrhunderts noch dominierenden Qualifikationsweg über die Universitäten in Frankreich und vor allem Italien. Dass man auch im ernestineschen Herzogtum Sachsen gern auf solche im Ausland qualifizierten Juristen zurückgriff, zeigt mein letztes Beispiel, Heinrich Husanus (1536-1587): in Eisenach geboren, Studium in Jena und 1553 in Wittenberg in der Artistenfakultät, dann Studium der Jurisprudenz in Bourges und Padua. Es folgten eine Tätigkeit am Reichskammergericht, 1561 bis 1562 eine juristische Professur in Jena, verbunden mit der Promotion zum Doktor der Rechte und dann eine Stelle als herzoglich sächsischer Rat. 1567 wechselte er in mecklenburgische Dienste und entwickelte für das Herzogtum eine Reihe von Rechts- und Prozessordnungen. ${ }^{32}$

Der Kreis meines Vortrags schließt sich mit einigen Bemerkungen zu seiner Hauptquelle, der Vitensammlung von Melchior Adam. Sammlungen von Biographien gesellschaftlicher Kollektive sind als prosopographische Quellen gerade für die Frühe Neuzeit meines Erachtens zu wenig erforscht. Mein Vortrag war der Versuch, typische Muster von Karriereverläufen und gesellschaftlichen Positionierungen von Theologen und Juristen des 16. Jahrhunderts weitestgehend aufgrund des zeitgenössischen biographischen Materials zu rekonstruieren. Die Biographien sind eine hervorragende Quellen für umfassende typologisierende und generalisierende Studien zu einer Vielzahl von Fragestellungen zu den Professoren der frühen Neuzeit: Karriereverläufe, Karriereförderung und Patronage, Gelehrtennetzwerke, Themenfelder wissenschaftlicher Arbeit, familiäre Verbindungen, schließlich das ganze Feld mentalitätsgeschichtlicher Fragestellungen: Verständnis von Lernen und Lehren, Berufsbilder, Persönlichkeitsprofile, Wertvorstellungen in Privat- und Berufsleben, schließlich die Ars moriendi. Zu bedenken ist dabei immer der Konstruktcharakter der Viten: Manches verrät mehr über den Biographienschreiber und seine Zeit als über die dargestellte Person.

32 Vgl. M. Adam, Vitae Germanorum Jureconsultorum et Politicorum, S. 130-132; Friedrich MerzBACHER, Art. Husanes, Heinrich, NDB 1023/1974, S. 80 f.; Walter KILly, Art. Husanus, Heinrich, DBE 5/1997, S. 232; Wilhelm Kühlmann, Art Husanus, Henricus, Killy Literaturlexikon 6/2009, S. 22; Hermann Wiegand, Art. Husanus, Henricus, Frühe Neuzeit in Deutschland. Literaturwissenschaftliches Verfasserlexikon 3/2014, S. 439-447. 


\section{Abkürzungen}

ADB Allgemeine Deutsche Biographie, 1875-1912

BBKL Biographisch-Bibliographisches Kirchenlexikon, 1975-2019

DBE Deutsche Biographische Enzyklopädie, 1995-2003

DBETh Deutsche Biographische Enzyklopädie der Theologie und der Kirchen, 2005

NDB Neue Deutsche Biographie, 1953-2016

RGG Religion in Geschichte und Gegenwart, ${ }^{4} 1998-2007$

TRE Theologische Realentyklopädie, 1977-2004

ZKG Zeitschrift für Kirchengeschichte, 1877-2019

\section{KLAUS-DIETER BEIMS}

\section{Profesoři a jejich vedlejší činnosti podle spisu Vitae (Životy) Melchiora Adama - kariéry mimo univerzitu}

\section{RESUMÉ}

Př́íspěvek se snaží rekonstruovat za pomoci biografií od Melchiora Adama (1575-1622) jako dobového pramene typické prvky kariérního postupu profesorů teologie a práva v 16. století a jejich mimouniverzitní aktivity. V raném období Reformace bylo charakteristické pro wittenbergské teology vedle univerzitní činnosti úzké osobní i institucionální propojení s církevními funkcemi. Byli využíváni jako církevní reformátoři a organizátoři i mimo Sasko. To je dokumentováno na př́ikladu Johanna Bugenhagena, který vedle své wittenbergské profesury teologie působil jako wittenbergský farář, superintendent saského kurfiřtství a církevní reformátor řady měst a teritorií. Také na univerzitě v Rostocku v polovině 16. století tamější obvykle dlouhodobě působící profesoři teologie byli vedle toho činní jako faráři, v úřadech řídících církev nebo jako církevně-političtí rádci knížat. Pro tento aspekt je dobrým př́íkladem kariéra Davida Chytraea. Profesoři právníci byli dosazováni do důležitých dvorských úřadů nebo na politické úkoly: jako knížecí kancléři nebo radové, členové nejvyššího soudu nebo dvorského soudu, do konsistoří rídících církev, resp. jako církevní radové, vyslanci na diplomatické mise, zástupci k řišskému komornímu soudu. Právníci př́ležitostně přijímali po domluvě s vlastním zeměpánem také rádcovské úkoly mimo své teritorium. Předpokladem pro pověření profesorů zvláště do vysokých kvalifikovaných pozic mimo univerzitu byla jejich promoce.

Dr. Klaus-Dieter Beims

Gymnasiallehrer a.D.

klausdieterbeims@web.de 